\title{
A Caged, Localizable Rhodamine Derivative for Superresolution Microscopy
}

\author{
Sambashiva Banala, ${ }^{\dagger, \S}$ Damien Maurel, ${ }^{\dagger, \S}$ Suliana Manley, ${ }^{\ddagger}$ and Kai Johnsson ${ }^{\dagger, *}$ \\ ${ }^{\dagger}$ Institute of Chemical Sciences and Engineering and ${ }^{\ddagger}$ Laboratory of Experimental Biophysics, École Polytechnique Fédérale de \\ Lausanne (EPFL), Lausanne, Switzerland
}

\section{Supporting Information}

ABSTRACT: A caged rhodamine 110 derivative for the specific labeling of SNAP-tag fusion proteins is introduced. The caged rhodamine 110 derivative permits the labeling of cell surface proteins in living cells and of intracellular proteins in fixed cells. The probe requires only a single caging group to

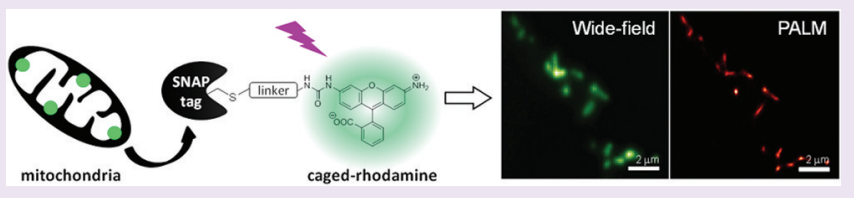
maintain the fluorophore in a non-fluorescent state and becomes highly fluorescent after uncaging. The high contrast ratio is confirmed both in bulk and at the single molecule level. This property, together with its high photon yield makes it an excellent dye for photoactivated localization microscopy (PALM), as we demonstrate here.

$\mathrm{T}$ The caging of a fluorophore is defined as its derivatization with a photocleavable group that converts the fluorophore into a non-fluorescent state. As the uncaging and resulting recovery of fluorescence through a light pulse can be achieved with high spatial and temporal resolution, caged fluorophores have become important tools in studying numerous biological processes, including cell lineage and protein trafficking. ${ }^{1,2}$ Another attractive application of caged fluorophores is in photoactivated localization microscopy (PALM), which permits imaging of cellular components with nanometer resolution. ${ }^{3}$ In PALM, sparse subsets of dye molecules are stochastically activated or uncaged, imaged, and bleached over the course of thousands of raw images. Since the density of activated molecules depends on the intensity of the activation light, we can ensure that molecules in each image are spatially wellseparated and thus can be localized by fitting to the pointspread function. ${ }^{4}$ The certainty with which a molecule can be localized depends inversely on the square-root of the number of photons collected, so the high photon yields of synthetic fluorophores offer a significant advantage over fluorescent proteins (FPs) in this respect. Furthermore, there are few options for good photoactivatable FPs in the green part of the spectrum for single-molecule-based imaging, and those that do exist suffer from high background and low contrast ratios. Caged fluorophores that could be coupled to selected proteins in living or fixed cells therefore have the potential to become important tools for superresolution microscopy. Caged versions of several fluorophores such as fluorescein and rhodamine have been reported, but caging of these fluorophores required the attachment of two photocleavable groups. ${ }^{1,2,5,6}$ The removal of two caging groups necessitates a longer irradiation time to recover the whole fluorescence from the illuminated sample. Moreover, attempts to selectively uncage only a subset of a population of caged fluorophores will mostly generate fluorophores where only one of the two caging groups has been removed. In the case of caged fluorescein and rhodamine derivatives, the partially uncaged fluorophore displays only relatively weak fluorescence compared to the fully uncaged fluorophore, and therefore this approach does not exploit the full potential of synthetic fluorophores for PALM. ${ }^{6}$ Caged versions of the fluorophore Tokyogreen with only one photocleavable group and of rhodamine with a 2-diazoketone (COCNN) caging group have been reported, ${ }^{7,8}$ but the specific coupling of these probes to individual proteins has not been described yet. Recently, a caged version of a dicyanomethylenedihydrofuran (DCDHF) fluorophore has been described that can be selectively coupled to Halo-tag fusion proteins. ${ }^{9}$ DCDHF was efficiently caged by replacing a crucial amino group with a photolabile azide; the probe is then uncaged by photoconversion of the azide back to the amino group. The potential of this caged DCDHF was demonstrated by performing PALM of Halo-tag fusion proteins in bacteria and mammalian cells. In summary, there is a generally acknowledged need for new localizable caged fluorophores.

Here we present a caged version of rhodamine $110\left(\mathrm{Rh}_{110}\right)$ that can be coupled to SNAP-tag fusion proteins and that is suitable for PALM (Figure 1). The large fluorescence increase upon uncaging and the brightness and stability of the SNAPtag-bound fluorophore make it an attractive probe for superresolution imaging and the study of dynamic cellular processes.

The design of our caged rhodamine probe 1 (BG-cRhod) was based on the observation that ureated derivatives of $\mathrm{Rh}_{110}$ retained much of the fluorescence of the parental compound. ${ }^{10}$ Particularly, the relative fluorescence intensity (a product of extinction coefficient and quantum yield) of the ureated derivative is $35 \%$ that of $\mathrm{Rh}_{110}$. The relative ease with which

Received: August 12, 2011

Accepted: October 25, 2011

Published: October 25, 2011 


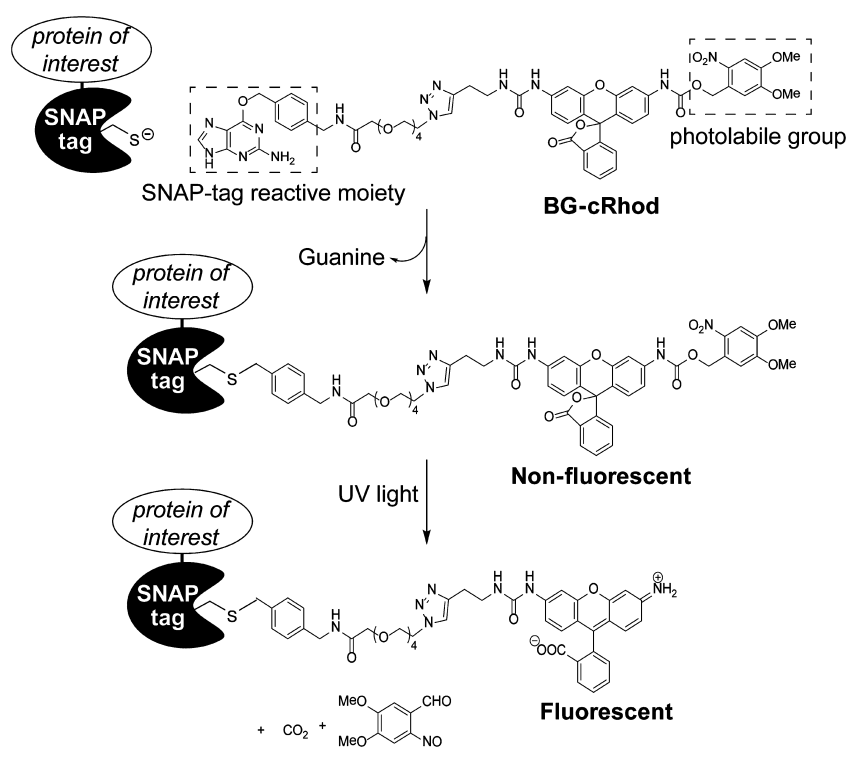

Figure 1. Design of the caged rhodamine BG-cRhod and its use for the labeling of SNAP-tag fusion proteins.

ureated $\mathrm{Rh}_{110}$ derivatives can be synthesized and their good spectroscopic properties have triggered the generation of a number of $\mathrm{Rh}_{110}$-based probes for various applications. ${ }^{11-13} \mathrm{On}$ the basis of these reports, we envisioned the synthesis of a caged $\mathrm{Rh}_{110}$ derivate for the reaction with SNAP-tag fusion proteins. SNAP-tag is a protein tag that reacts with $\mathrm{O}^{6}$ benzylguanine (BG) derivatives carrying different probes in vitro and in vivo. ${ }^{14,15}$ In BG-cRhod, a BG is attached via a urea linkage to $\mathrm{Rh}_{110}$, while the attachment of a photocleavable 4,5dimethoxy-2-nitrobenzyl group (DMNB) to the other amino group of $\mathrm{Rh}_{110}$ would force the probe in its non-fluorescent lactone configuration (Figure 1). We anticipated that SNAP-tag fusion proteins could be labeled with BG-cRhod and subsequently uncaged through irradiation with UV light.

Starting from commercially available $\mathrm{Rh}_{110}$, BG-cRhod was synthesized in five steps in a total yield of $4.3 \%$ (Figure 2, panel A). The fluorescence properties of BG-cRhod were studied in vitro before and after reaction with SNAP-tag. First, we confirmed by HPLC and mass spectroscopy that the photolysis of BG-cRhod generated the corresponding $B G \mathrm{Rh}_{110}$ derivative BG-Rhod (Supplementary Figure 1). As expected, BG-cRhod is non-fluorescent $\left(\Phi_{\mathrm{fl}}<0.001\right)$ and became fluorescent upon irradiation with $365 \mathrm{~nm}$ light, although the fluorescence quantum yield of BG-cRhod was relatively low $\left(\Phi_{\mathrm{fl}}=0.074\right)$ (Figure 2, panel B). We then demonstrated that BG-cRhod permits the labeling of SNAP-tag with cRhod (Supplementary Figures 3 and 4). As observed on BG-cRhod, SNAP-tag-bound cRhod is virtually non-fluorescent $\left(\Phi_{\mathrm{fl}}<0.002\right)$. However, the fluorescence quantum yield of uncaged SNAP-tag-bound cRhod increased to 0.36 , which is about 5 times higher than that of the uncaged free substrate (Figure 2, panel B). The observed increase of fluorescence upon uncaging of SNAP-tagbound cRhod is at least 200 -fold. We assume that the background fluorescence observed prior to uncaging is most likely due to the presence of small amounts of already uncaged BG-cRhod. The low fluorescence quantum yield of BG-cRhod is most likely due to quenching through benzylguanine. ${ }^{16,17}$ Similar behavior has already been observed for other fluorescent BG derivatives used for SNAP-tag labeling. ${ }^{18-20}$ The observed quenching in uncaged BG-cRhod relative to that
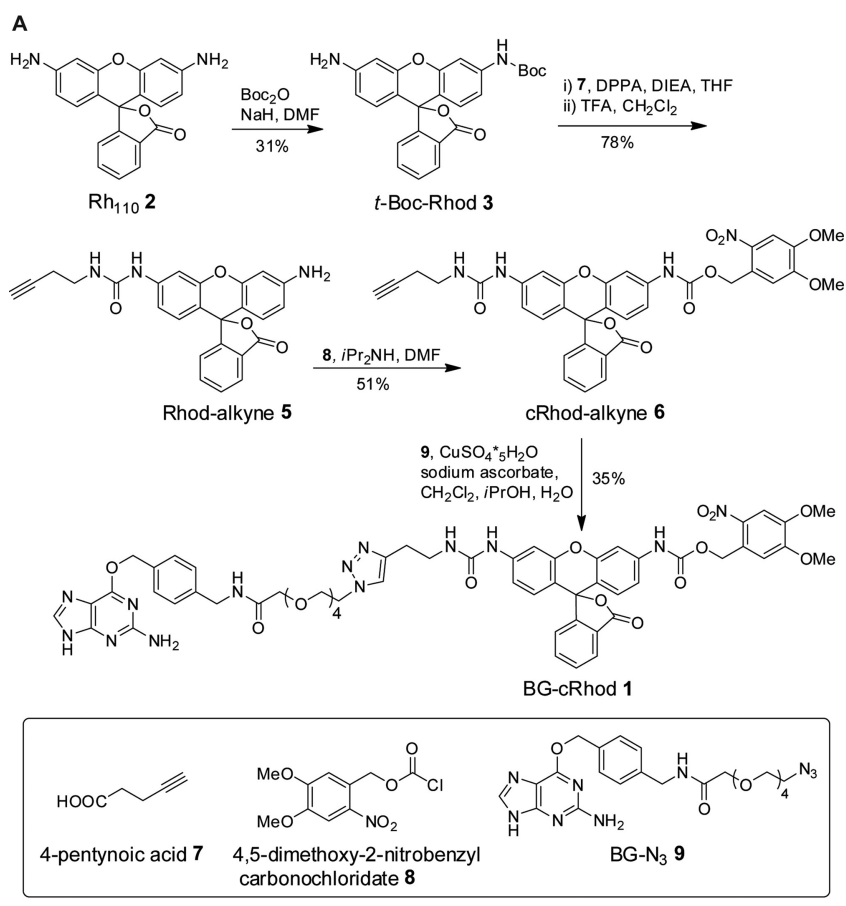

B

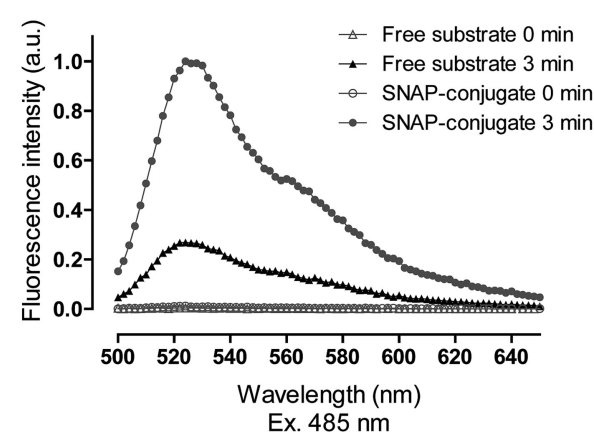

Figure 2. (A) Synthesis of BG-cRhod. (B) In vitro characterization of BG-cRhod. Fluorescence increase of free BG-cRhod (triangles) and its SNAP-tag conjugate (circles) before (open) and after (filled) uncaging. An enlargement of the fluorescence spectrum of caged BG-cRhod and caged SNAP-tag-bound cRhod is shown in Supporting Information. $\mathrm{Boc}_{2} \mathrm{O}=$ di-tert-butyldicarbonate, DIEA $=\mathrm{N}, \mathrm{N}$ diisopropylethylamine, DPPA = diphenylphosphoryl azide, $i \operatorname{Pr}_{2} \mathrm{NH}=$ diisopropylamine, $\mathrm{TFA}=$ trifluoroacetic acid, DMF = dimethylformamide, $\mathrm{THF}=$ tetrahydrofuran.

of SNAP-tag-bound uncaged cRhod is an advantageous feature of our probe because it reduces potential background fluorescence from unreacted, uncaged probe.

We then investigated the use of BG-cRhod for the labeling of cell surface receptors and subsequent uncaging of labeled protein. We chose the $\beta$-adrenergic receptor $\beta 2 \mathrm{AR}$ as a model protein as its SNAP-tag fusion has previously been shown to be functional. ${ }^{21}$ HEK293T cells were transiently transfected with a plasmid for expression of SNAP- $\beta 2 A R$ and consecutively labeled with BG-cRhod $(0.3 \mu \mathrm{M}, 1 \mathrm{~h})$ and BG-Cy5 $(2 \mu \mathrm{M}$, $10 \mathrm{~min})$. Simultaneous labeling with BG-Cy5 was performed to identify transfected cells expressing SNAP- $\beta 2 A R$ prior to uncaging. Using a $488 \mathrm{~nm}$ laser for excitation, no significant fluorescence of BG-cRhod-labeled SNAP- $\beta 2 A R$ could be detected prior to uncaging (Figure 3, panel A). However, irradiation with a $405 \mathrm{~nm}$ laser resulted in uncaging of labeled SNAP- $\beta 2 A R$, as demonstrated by the strong fluorescence signal observed at the plasma membrane of transfected cells (Figure 3, 
a
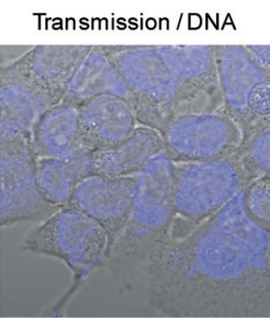

b

Transmission /DNA

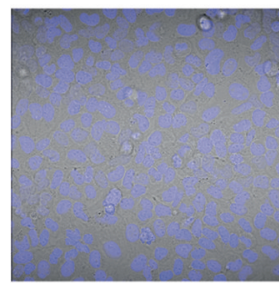

C

Transmission / DNA

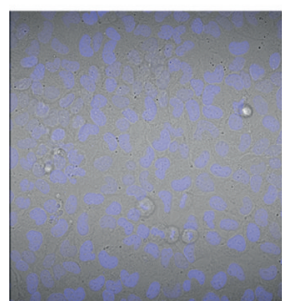

BG-Cy5

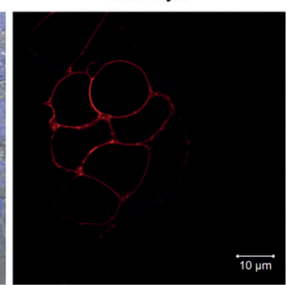

$\overline{10 \mu \mathrm{m}}$

BG-Cy5

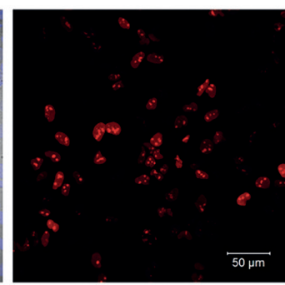

$\overline{50 \mu \mathrm{m}}$

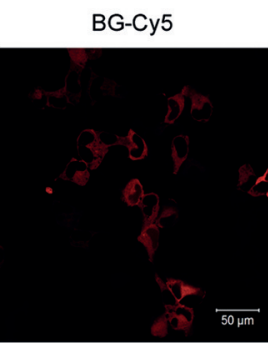

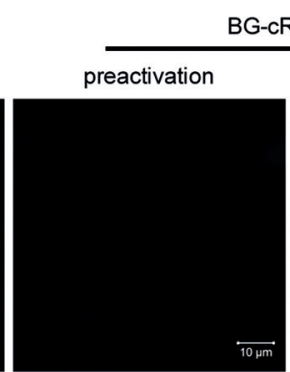

postactivation

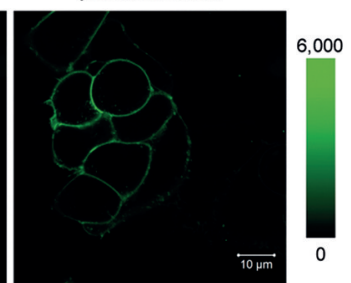

BG-cRhod

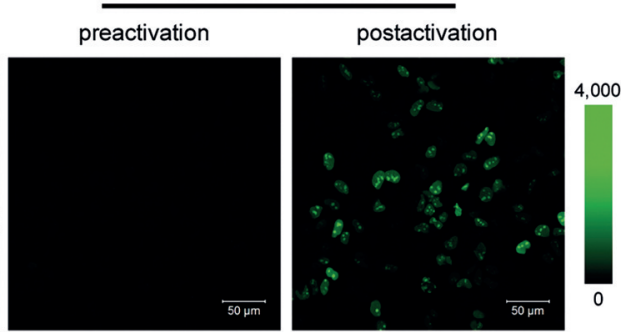

BG-cRhod

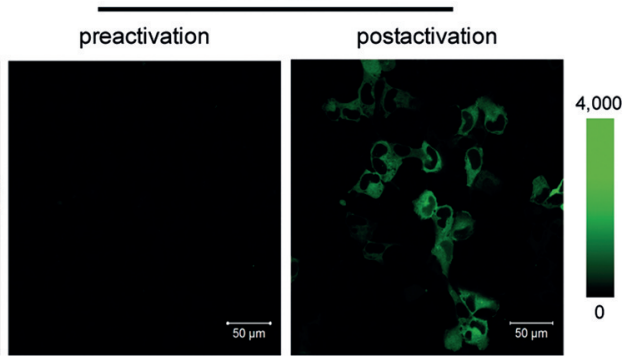

Figure 3. (A) Confocal fluorescence images of living HEK293T cells expressing SNAP- $\beta 2$ adrenergic receptor. Transiently expressed SNAP- $\beta 2$ AR was labeled with BG-cRhod (green) and then with BG-Cy5 (red). Nuclear DNA staining with Hoechst 33342 was used as a reference (blue). Images were taken before and after UV irradiation. (B, C) Confocal fluorescence images of fixed U2OS cells expressing SNAP-NLS and SNAP-MEK1. (B) Transiently expressed SNAP-NLS (nucleus) and (C) SNAP-MEK1 (cytoplasm) were labeled with the SNAP-tag substrates BG-cRhod (green) and then with BG-Cy5 (red). Nuclear DNA staining with Hoechst 33342 was used as a reference (blue). Images were taken before and after uncaging at $405 \mathrm{~nm}$. Cells were fixed with paraformaldehyde prior to staining.

panel A). The possibility to uncage BG-cRhod-labeled proteins with a $405 \mathrm{~nm}$ laser is important for practical applications as this laser line is found on most confocal microscopes and illumination at $405 \mathrm{~nm}$ is less phototoxic than illumination with UV light.

We then tested if BG-cRhod permits the labeling of intracellular SNAP-tag fusion proteins. For these experiments, a fusion protein of SNAP-tag with the kinase MEK1 (SNAPMEK1) was chosen as an example of a cytosolic protein and transiently expressed in U2OS cells. Although labeling of SNAP-MEK1 with the cell-permeable fluorescent SNAP-tag substrate TMR-star was achieved, no significant labeling with BG-cRhod could be observed (Supplementary Figure 5). The failure to label intracellular SNAP-tag fusion proteins with BGcRhod in living cells limits the application range of this substrate. However, the labeling of intracellular SNAP-tag fusion proteins with BG-cRhod could be achieved with invasive approaches such as bead-loading, microinjection or electroporation; all of these approaches have been previously been shown to permit the labeling of SNAP-tag fusion proteins with cell-impermeable substrates. ${ }^{2-24}$

Superresolution imaging using point-based localization (PALM, STORM, etc.) for the elucidation of biological structures is generally performed on fixed cells. ${ }^{25,26} \mathrm{We}$ therefore investigated if the selective labeling of SNAP-tag fusion proteins with BG-cRhod can be achieved after fixation. To this end, we performed labeling experiments with BGcRhod on fixed U2OS cells expressing SNAP-tag fusion proteins localized either in the nucleus (SNAP-NLS) or in the cytoplasm (SNAP-MEK1) (Figure 3, panels B and C). Cells were fixed with paraformaldehyde and consecutively labeled with BG-cRhod $(0.3 \mu \mathrm{M}, 1 \mathrm{~h})$ and then BG-Cy5 $(2 \mu \mathrm{M}, 10$ $\mathrm{min})$ to facilitate the identification of transiently transfected cells. After labeling with BG-cRhod and uncaging with a 405 $\mathrm{nm}$ laser, cells showed the expected localized fluorescence signals. In contrast, no significant fluorescence was detected prior to photoactivation. These experiments demonstrate that BG-cRhod is suitable for the labeling of SNAP-tag fusion proteins in fixed cells and suggest that the probe is suitable for PALM.

For PALM imaging, we used BG-cRhod to label SNAP-tag fusion proteins localized to mitochondria (Mito-SNAP). U2OS cells expressing Mito-SNAP were fixed with paraformaldehyde and labeled with BG-cRhod $(0.3 \mu \mathrm{M}, 2 \mathrm{~h})$. Before PALM imaging, cells were exposed to a high level of $488 \mathrm{~nm}$ light to photobleach any previously uncaged molecules. The sum of all raw images corresponds to a time-integrated epifluorescence image (Figure 4, panel A), which is shown for comparison with the rendered super-resolution PALM image (Figure 4, panel 

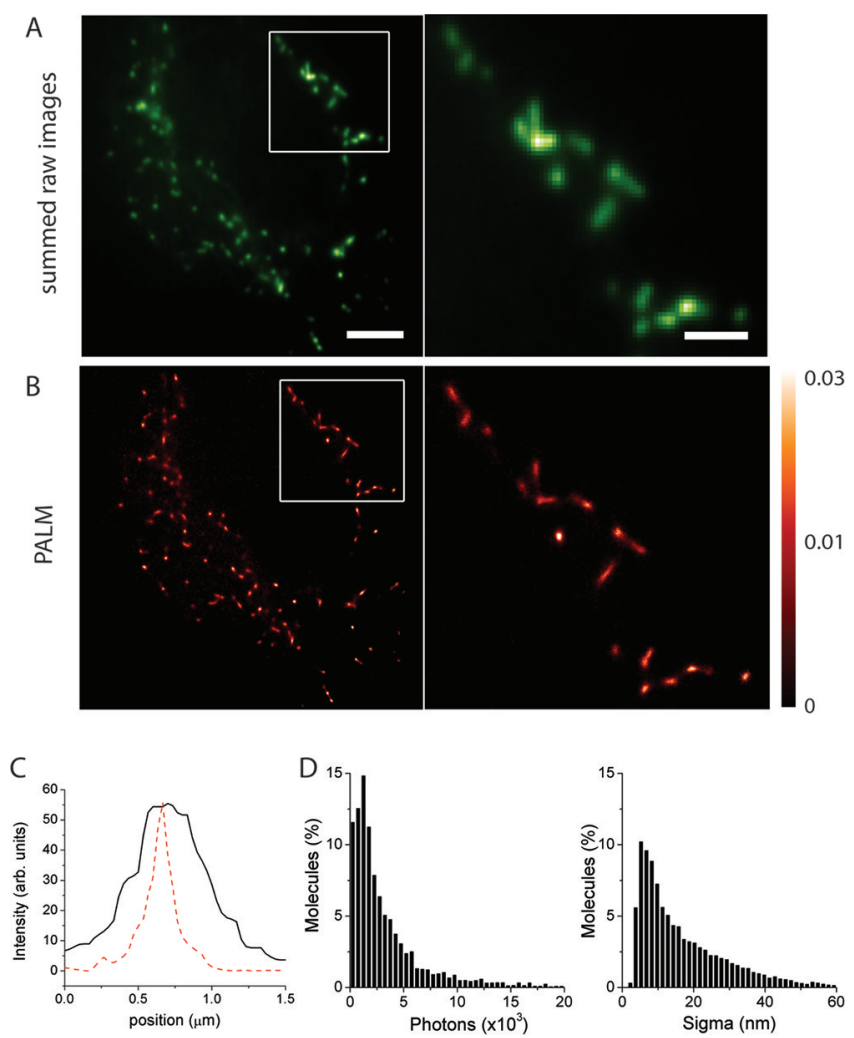

Figure 4. (A) Summed raw images of fixed U2OS cells expressing SNAP-Mito (mitochondria). Quasi-stable cells expressing a mitochondrial targeting sequence were labeled with BG-cRhod, and a stack of 5000 images was collected with sparse activation using a $405 \mathrm{~nm}$ laser and then summed to create the equivalent of an epi-fluorescence image. (B) PALM images. The same stack of images was processed for PALM. Color bar corresponds to the probability density of finding a molecule in units of molecules per $\mathrm{nm}^{2}$. Number of molecules: 142,433 (left), 16,960 (right). (C) Intensity profiles across a single mitochondria were analyzed to extract the full width half max from a Gaussian fit for summed raw (solid line, fwhm $=517 \mathrm{~nm}$ ) and PALM (dashed line, fwhm $=135 \mathrm{~nm}$ ) images. (D) Histograms of the number of photons and molecular localization uncertainty. The molecules shown in panel B were analyzed for their photon yield (mean $=3488$; median $=2027)$ and localization uncertainties, $\sigma($ mean $=16 \mathrm{~nm}$, median $=11.7 \mathrm{~nm}$ ). Scale bar (left) is $5 \mu \mathrm{m}$, scale bar (right, zoom) is $2 \mu \mathrm{m}$.

B). As expected, features that are obscured in wide-field imaging are sharply resolved in the PALM image. We used the information from the raw data to obtain statistics on molecular photon yields and localization precision. In comparison with the best red FPs or PA-GFP, which give on average 300-800 photons per molecule (ref 27 and Supplementary Figure S7), we found a mean value of 3488 photons/molecule, an increase in photon yields of a factor of $\sim 5-10$. This increased molecular brightness is translated into an improvement in localization; because there is a rather long tail in the distribution this is demonstrated by the median value of uncertainty in position, which is $11.7 \mathrm{~nm}$, a factor of $\sim 2$ better than for the red FPs. The mode of the distribution is even lower, $6.1 \mathrm{~nm}$, a factor of $\sim 3$ better than for the red FPs. Thus, cRhod is an excellent label for PALM imaging in general.

Stochastic optical reconstruction microscopy (STORM), direct STORM (dSTORM), and "ground state depletion microscopy followed by individual molecule return" (GSDIM) are alternative approaches toward superresolution microscopy. ${ }^{28-30}$ These methods rely on synthetic fluorophores subjected to reduction-oxidation or triplet-state cycling to induce blinking and therefore temporally separated signals as required by point-localization superresolution microscopy. Compared to PALM, these approaches have been able to exploit the higher photon counts available from synthetic dyes, resulting in higher molecular localization precision. However, for high densities of molecules, it can be difficult to fully initialize the system into the dark state. Furthermore, it is not straightforward to control the rate of molecules cycling, to maintain their temporal separation. Caged dyes such as cRhod provide an advantage in that their initial state is largely the caged one, with the possibility of easily photobleaching any small uncaged population. Moreover, their uncaging is wellcontrolled as a function of the intensity of the UV uncaging light. Another interesting aspect of cRhod for future practical applications is the possibility to use it in combination with red FPs or synthetic photoswitchable probes for multicolor imaging. In light of these general considerations and the favorable spectroscopic properties of $\mathrm{cRhod}$, we expect that this dye will become a useful addition to the already available dyes for superresolution microscopy.

\section{ASSOCIATED CONTENT}

\section{S Supporting Information}

Detailed experimental procedures and characterization of compounds. This material is available free of charge via the Internet at http://pubs.acs.org.

\section{AUTHOR INFORMATION}

\section{Corresponding Author}

*E-mail: kai.johnsson@epfl.ch.

\section{Author Contributions}

$\S_{\text {}}$ These authors contributed equally to this work.

\section{ACKNOWLEDGMENTS}

This work was supported by the Swiss National Science Foundation and partially supported by funding from the European Research Council under the EC FP7/ERC grant agreement $n^{\circ}$ 243016-PALMassembly. The authors thank Grazvydas Lukinavicius and Carine Ben-Adiba for technical assistance.

\section{REFERENCES}

(1) Marriott, G., and Ottl, J. (1998) Synthesis and applications of heterobifunctional photocleavable cross-linking reagents. Methods Enzymol. 291, 155-175.

(2) Mitchison, T. J., Sawin, K. E., Theriot, J. A., Gee, K., and Mallavarapu, A. (1998) Caged fluorescent probes. Methods Enzymol. 291, 63-78.

(3) Fernandez-Suarez, M., and Ting, A. Y. (2008) Fluorescent probes for super-resolution imaging in living cells. Nat. Rev. Mol. Cell Biol. 9, 929-943.

(4) Thompson, R. E., Larson, D. R., and Webb, W. W. (2002) Precise nanometer localization analysis for individual fluorescent probes. Biophys. J. 82, 2775-2783.

(5) Gee, K. R., Weinberg, E. S., and Kozlowski, D. J. (2001) Caged Q-rhodamine dextran: a new photoactivated fluorescent tracer. Bioorg. Med. Chem. Lett. 11, 2181-2183.

(6) Wysocki, L. M., Grimm, J. B., Tkachuk, A. N., Brown, T. A., Betzig, E., Lavis, L. D. (2011) Facile and general synthesis of photoactivatable xanthene dyes, Angew. Chem., Int. Ed. Epub ahead of print, DOI: 10.1002/anie.201104571. 
(7) Kobayashi, T., Urano, Y., Kamiya, M., Ueno, T., Kojima, H., and Nagano, T. (2007) Highly activatable and rapidly releasable caged fluorescein derivatives. J. Am. Chem. Soc. 129, 6696-6697.

(8) Belov, V. N., Wurm, C. A., Boyarskiy, V. P., Jakobs, S., and Hell, S. W. (2010) Rhodamines NN: A novel class of caged fluorescent dyes. Angew. Chem., Int. Ed. 49, 3520-3523.

(9) Lee, H. L., Lord, S. J., Iwanaga, S., Zhan, K., Xie, H., Williams, J. C., Wang, H., Bowman, G. R., Goley, E. D., Shapiro, L., Twieg, R. J., Rao, J., and Moerner, W. E. (2010) Superresolution imaging of targeted proteins in fixed and living cells using photoactivatable organic fluorophores. J. Am. Chem. Soc. 132, 15099-15101.

(10) Lavis, L. D., Chao, T. Y., and Raines, R. T. (2006) Fluorogenic label for biomolecular imaging. ACS Chem. Biol. 1, 252-260.

(11) Yatzeck, M. M., Lavis, L. D., Chao, T. Y., Chandran, S. S., and Raines, R. T. (2008) A highly sensitive fluorogenic probe for cytochrome P450 activity in live cells. Bioorg. Med. Chem. Lett. 18, $5864-5866$.

(12) Pianowski, Z., Gorska, K., Oswald, L., Merten, C. A., and Winssinger, N. (2009) Imaging of mRNA in live cells using nucleic acid-templated reduction of azidorhodamine probes. J. Am. Chem. Soc. 131, 6492-6497.

(13) Mangold, S. L., Carpenter, R. T., and Kiessling, L. L. (2008) Synthesis of fluorogenic polymers for visualizing cellular internalization. Org. Lett. 10, 2997-3000.

(14) Keppler, A., Gendreizig, S., Gronemeyer, T., Pick, H., Vogel, H., and Johnsson, K. (2003) A general method for the covalent labeling of fusion proteins with small molecules in vivo. Nat. Biotechnol. 21, 8689.

(15) Keppler, A., Pick, H., Arrivoli, C., Vogel, H., and Johnsson, K. (2004) Labeling of fusion proteins with synthetic fluorophores in live cells. Proc. Natl. Acad. Sci. U.S.A. 101, 9955-9959.

(16) Seidel, C. A. M., Schulz, A., and Sauer, M. H. M. (1996) Nucleobase-specific quenching of fluorescent dyes 0.1. Nucleobase one-electron redox potentials and their correlation with static and dynamic quenching efficiencies. J. Phys. Chem. 100, 5541-5553.

(17) Crockett, A. O., and Wittwer, C. T. (2001) Fluorescein-labeled oligonucleotides for real-time pcr: using the inherent quenching of deoxyguanosine nucleotides. Anal. Biochem. 290, 89-97.

(18) Tomat, E., Nolan, E. M., Jaworski, J., and Lippard, S. J. (2008) Organelle-specific zinc detection using zinpyr-labeled fusion proteins in live cells. J. Am. Chem. Soc. 130, 15776-15777.

(19) Bannwarth, M., Correa, I. R., Sztretye, M., Pouvreau, S., Fellay, C., Aebischer, A., Royer, L., Rois, E., and Johnsson, K. (2009) Indo-1 derivatives for local calcium sensing. ACS Chem. Biol. 4, 179-190.

(20) Stohr, K., Siegberg, D., Ehrhard, T., Lymperopoulos, K., Oz, S., Schulmeister, S., Pfeifer, A. C., Bachmann, J., Klingmuller, U., Sourjik, V., and Herten, D. P. (2010) Quenched substrates for live-cell labeling of SNAP-tagged fusion proteins with improved fluorescent background. Anal. Chem. 82, 8186-8193.

(21) Maurel, D., Comps-Agrar, L., Brock, C., Rives, M. L., Bourrier, E., Ayoub, M. A., Bazin, H., Tinel, N., Durroux, T., Prezeau, L., Trinquet, E., and Pin, J. P. (2008) Cell-surface protein-protein interaction analysis with time-resolved FRET and snap-tag technologies: application to GPCR oligomerization. Nat. Methods 5, 561-567.

(22) Maurel, D., Banala, S., Laroche, T., and Johnsson, K. (2010) Photoactivatable and photoconvertible fluorescent probes for protein labeling. ACS Chem. Biol. 5, 507-516.

(23) Keppler, A., Arrivoli, C., Sironi, L., and Ellenberg, J. (2006) Fluorophores for live cell imaging of AGT fusion proteins across the visible spectrum. Biotechniques 41, 167-170, , 172, 174-165.

(24) Jones, S. A., Shim, S. H., He, J., and Zhuang, X. (2011) Fast, three-dimensional super-resolution imaging of live cells. Nat. Methods $8,499-505$.

(25) Shroff, H., Galbraith, C. G., Galbraith, J. A., White, H., Gillette, J., Olenych, S., Davidson, M. W., and Betzig, E. (2007) Dual-color superresolution imaging of genetically expressed probes within individual adhesion complexes. Proc. Natl. Acad. Sci. U.S.A. 104, 20308-20313.
(26) Huang, B., Wang, W., Bates, M., and Zhuang, X. (2008) Threedimensional super-resolution imaging by stochastic optical reconstruction microscopy. Science 319, 810-813.

(27) Subach, F. V., Patterson, G. H., Manley, S., Gillette, J. M., Lippincott-Schwartz, J., and Verkhusha, V. V. (2009) Photoactivatable mCherry for high-resolution two-color fluorescence microscopy. Nat. Methods 6, 153-159.

(28) Rust, M. J., Bates, M., and Zhuang, X. (2006) Sub-diffractionlimit imaging by stochastic optical reconstruction microscopy (STORM). Nat. Methods 3, 793-795.

(29) Heilemann, M., van de Linde, S., Schuttpelz, M., Kasper, R. Seefeldt, B., Mukherjee, A., Tinnefeld, P., and Sauer, M. (2008) Subdiffraction-resolution fluorescence imaging with conventional fluorescent probes. Angew. Chem., Int. Ed. 47, 6172-6176.

(30) Folling, J., Bossi, M., Bock, H., Medda, R., Wurm, C. A., Hein, B., Jakobs, S., Eggeling, C., and Hell, S. W. (2008) Fluorescence nanoscopy by ground-state depletion and single-molecule return. Nat. Methods 5, 943-945. 